\title{
Robust Adaptive Control of an Offshore Ocean Thermal Energy Conversion System
}

\author{
Xiuyu He, Student Member, IEEE, Wei He, Senior Member, IEEE, Yingru Liu, Yiheng Wang, Guang Li, \\ Yu Wang
}

\begin{abstract}
Boundary control strategy is developed to analyze the vibration problem of the offshore ocean thermal energy conversion (OTEC) system as well as to constrain the bottom tension and top motion. To provide an accurate dynamic behavior for the OTEC system, this distributed parameter system is modeled and formulated with a governing equation and boundary conditions (PDE-ODEs model). Two robust adaptive boundary controllers are designed and disposed at the endpoints of the system, and the stability of the controlled system under unknown disturbances is achieved. After selecting the relevant parameters appropriately, the offset of the offshore OTEC system can be suppressed to equilibrium position. Finally, the effectiveness of the proposed control is illustrated by simulation.
\end{abstract}

Index Terms-Offshore OTEC system, Vibration control, Distributed parameter system, Robust adaptive control.

\section{INTRODUCTION}

Ocean energy is a class of promising renewable energy resources (e.g. wind, solar, wave, tide, thermal energy, etc.) and has attracted increasing attention [1]. Ocean thermal energy provides a vast, persistent and available renewable energy resource and the ocean thermal energy conversion uses the temperature difference between deep cold seawater $\left(4^{\circ} \mathrm{C} \sim 7^{\circ} \mathrm{C}\right)$ in $500 \mathrm{~m} \sim 1000 \mathrm{~m}$ and shallow warm seawater $\left(25^{\circ} \mathrm{C} \sim 28^{\circ} \mathrm{C}\right)$ to derive a heat engine and generate the electric energy further [2], [3], [4]. Based on principles of Rankine cycle, it utilizes the temperature difference between cool deep water and warm shallow seawater to run a turbine which can create electric power, then transport the electricity to onshore power grid through undersea cables [5]. There are mainly two types of the offshore OTEC system: the closedcycle and open-cycle systems, where, some low boiling points fluids are used to power the electricity generator for the closed-cycle one [6], and the open-cycle system utilizes the vapor generated by the seawater [7]. Compared with other renewable energy techniques, there are three major advantages of offshore OTEC technology. First, it offers a sustainable electricity production method that can run day and night and year-round. Second, offshore OTEC system can generate other

X. He and W. He are with School of Automation and Electrical Engineering, Institute of Artificial Intelligence and Key Laboratory of Knowledge Automation for Industrial Processes, Ministry of Education, University of Science and Technology Beijing, Beijing 100083, China. (e-mail: weihe@ieee.org).

Y. Liu is with Department of Electrical and Computer Engineering, Strony Brook, New York, USA, Y. Wang is with Department of Electrical and Computer Engineering, Missouri University of Science and Technology, Rolla, MO, USA, G. Li is with Queen Mary, University of London, Mile End Road, London E1 4NS, UK, and Y. Wang is with Institute of Automation, Chinese Academy of Sciences, Beijing 100190, China.

This work was supported by the National Natural Science Foundation of China under Grant 61522302, 61761130080, 61520106009, and the Beijing Science and Technology Project under Grant Z181100003118006. beneficial byproducts, such as fresh water, nutrients and so on. Finally, this method can be applied in any tropical areas, which contain vast ocean thermal resource, to make them more self-sufficient in power supply [8], [9]. For these reasons, the OTEC technique is quite prospective and will be widely used in the near future.

A large amount of research results on offshore OTEC system have been presented [10], [11], [12], [13]. In [12], the authors analyze the dynamic characteristic and stability of the offshore solar-concentrated OTEC system. In [13], a nonlinear separation control structure is designed to improve the operation of the OTEC power cycle. In [14], a designed model of the evaporator and separator in an OTEC plant using the Uehara cycle is constructed to realize liquid level control. In [15], a secondary power loop is introduced to reuse the wasted heat of the main power cycle and the effectiveness of this technique is demonstrated. In a nuclear power plant, an alternative structure is discussed for replacing surface seawater with condenser effluent in [16].

As a crucial component in OTEC system, pipe is used to transport the deep cold seawater and this deep pipeline is subject to the external ocean disturbances and generates the vibration problem because of the large length of the pipe. It will weaken the performance of the OTEC system, even destroy this flexible structure. Recent researches about the flexible structures have been developed [17], [18], [19], [20], [21]. In [22], the authors studied the vibration problem and the stochastic disturbances for a flexible marine riser system. In [23], a flexible moving rod was investigated in twodimensional space and vibration control was designed to track the target positions rejection. Up to now, little attention has been paid to suppress the vibration of the long pipe which links the ballast and the platform on the ocean surface. Thus, some practical control methods should be designed and applied in this system to solve the problem.

For the OTEC system, the flexible pipe is a distributed parameter system due to the large span and partial differential equations are used to describe this DPS in mathematical [24], [25], [26], [27], [28], [29], [30]. Many control methods have been developed for DPS [31], [32], [33], [34], [35]. In [31], the authors developed a force control method to reduce the vibration and to drive the Timeoshenko arm track a desired position. In [36], backstepping method are used into a DPS based on wave PDE dynamics with a moving free boundary. In [37], UDE technique was used to develop boundary control for handling the uncertainty and disturbances in an unstable parabolic system. In [35], [38], the authors developed iterative learning control method for stabilizing the distributed parameter systems. 
Boundary control is a particular method for DPS, especially for the flexible systems because of the controller constitution and implemental position [39], [40], [41], [42], [43], [44]. The authors bring in the adaptive vibration control method to reduce the vibration of axially translating beam in [45]. In [46], a simple boundary cooperative control is used to drive the two one-link flexible arms completing grasping task. In [47], a boundary feedback control is considered for a firstorder, nonlinearly coupled Hyperbolic Lotka-Volterra systems. In [48], an effective boundary control is brought in the flexible marine riser to achieve top tension constraint.

In the real environment, there exist many parameters in the system and they have great influence on the dynamics of the system and control performance [49], [50], [51]. Besides, the output limitation problem which has influence on the security of the system is common in the actual environment [52], [53], [54], [55], [56]. The logarithmic barrier term is an effective method to constraint the desired limited signal. An adaptive control is applied at the endpoints of the pipe to deal with the vibration problem under the external disturbances in this paper. In addition, the output constraint problems are solved and the stability of the system is analyzed simultaneously. The innovations of this paper include:

(1) For the ocean thermal energy conversion system, the distributed tension is considered and the nonlinear PDEsODEs dynamic model of this system is established.

(2) Adaptive vibration control scheme is developed with the disturbance observers to stabilize the uncertain OTEC system and reject the ocean disturbances. The states of the system are convergent with uniformly bound, namely, the vessel is positioned near the original position and the deformation of the pipe is reduced.

The paper is organized as follows: The dynamic analysis and modeling for the offshore OTEC system are completed in Section II, and the pipe of the OTEC system is derived and described with a hybrid PDE-ODEs model. Besides, some relevant preliminaries are given for the subsequent development. In Section III, the control schemes are proposed and the controlled system is proven to be stable. In Section IV, the theoretical proof of the designed control strategy is verified by numerical experiment simulation given. Finally, we make the conclusion about this paper in Section V.

\section{Problem Formulation and Modeling for OTEC SYSTEM}

In order to develop an effective vibration control for the OTEC system, the dynamical characteristic of this system should be analyzed under the external ocean disturbances. In this section, we give the problem formulation to describe the OTEC system and the infinite dimensional dynamic model is established by using the Hamilton principle. Shown in Fig. 1, a typical offshore OTEC system is consist of three significant parts: a vessel, a flexible pipe serving as cold water intake and a ballast linked with the pipe to minimize the transverse motion at the bottom of the pipe. The cold water will be driven into a condenser, and warm water from the shallow ocean is driven into an evaporator. Then the power cycle between the condenser and evaporator will promote a turbine to generate electric energy. In this paper, we only consider the motion in the horizontal direction. Thus, the motion and effects of disturbances are neglected in vertical plane.

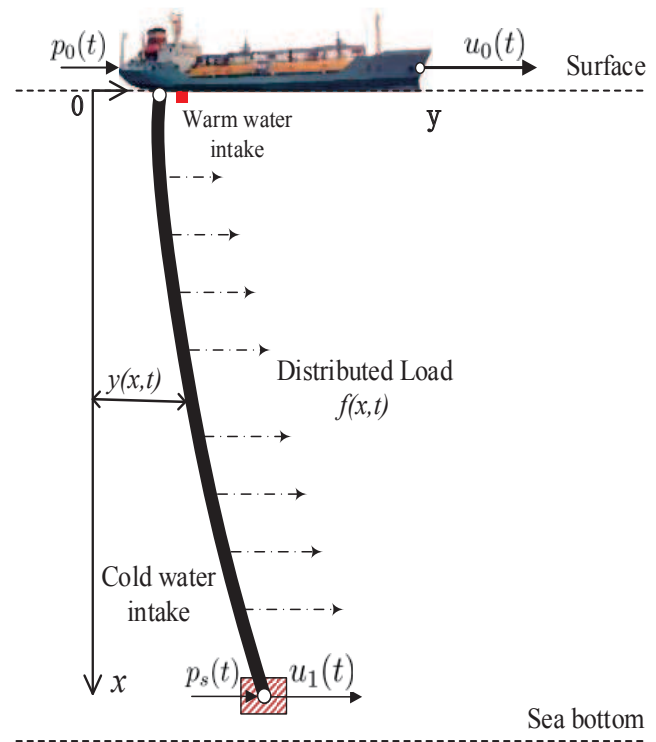

Fig. 1. The structure of an offshore OTEC plant

For clarity, the notations $(\dot{*})=\frac{\partial(*)}{\partial t},(\ddot{*})=\frac{\partial^{2}(*)}{\partial t^{2}}(*)^{\prime}=$ $\frac{\partial(*)}{\partial x},(*)^{\prime \prime}=\frac{\partial^{2}(*)}{\partial x^{2}},(*)^{\prime \prime \prime}=\frac{\partial^{3}(*)}{\partial x^{3}}$, and $(*)^{\prime \prime \prime \prime}=\frac{\partial^{4}(*)}{\partial x^{4}}$, are adopted throughout the paper.

\section{A. Dynamics of the OTEC system}

In this paper, Hamilton's principle is used to analyze the dynamics based on the energy functions of the system, which is given as [57]:

$$
\int_{t_{1}}^{t_{2}} \delta\left[E_{k}(t)-E_{p}(t)+W(t)\right] d t=0
$$

where the time instants $t_{1}$ and $t_{2}$ are given to define the operating interval $t \in\left(t_{1}, t_{2}\right)$, and $\delta$ is variational operator.

In the OTEC system, the floater is regarded as a rigid body and the cole water pipe's rotary inertia is neglected. We obtain the kinetic energy as

$$
E_{k}(t)=\frac{M_{s}}{2} \dot{y}^{2}(L, t)+\frac{\rho}{2} \int_{0}^{L} \dot{y}^{2}(x, t) d x+\frac{M_{0}}{2} \dot{y}^{2}(0, t)
$$

where $M_{s}$ and $M_{0}$ denote the lump mass of the ballast and the vessel and $\rho$ represents the distributed mass of the pipe. $y(x, t)$ denotes the displacement of the flexible pipe at the position $x$ for time $t$. The transverse displacements $y(L, t)$ and $y(0, t)$ describe the movement of the ballast under the deep water and the vessel in the surface. Three terms of the kinetic energy equation represent the kinetic energy of ballast, cold water pipe and the vessel, respectively.

The potential energy is presented as

$$
E_{p}(t)=\frac{E I}{2} \int_{0}^{L}\left[y^{\prime \prime}(x, t)\right]^{2} d x+\frac{1}{2} \int_{0}^{L} T(x, t)\left[y^{\prime}(x, t)\right]^{2} d x
$$

where $E I$ describes the bending stiffness and the distributed tension $T(x, t)$ of the pipe related with the initial tension $T_{0}(x)$ 
and the nonlinear nonnegative elastic modulus $\lambda(x)$ [58] is defined as

$$
T(x, t)=T_{0}(x)+\lambda(x)\left[y^{\prime}(x, t)\right]^{2}
$$

The virtual works done by the damping on the pipe, vessel and ballast, the boundary control forces and the environmental disturbances $f(x, t), p_{s}(t)$ and $p_{0}(t)$, are defined as

$$
\begin{aligned}
\delta W_{d}(t)= & -\int_{0}^{L} c \dot{y}(x, t) \delta y(x, t) d x-d_{s} \dot{y}(L, t) \delta y(L, t) \\
& -d_{0} \dot{y}(0, t) \delta y(0, t) \\
\delta W_{m}(t)= & u_{1}(t) \delta y(L, t)+u_{0}(t) \delta y(0, t) \\
\delta W_{f}(t)= & \int_{0}^{L} f(x, t) \delta y(x, t) d x+p_{s}(t) \delta y(L, t) \\
& +p_{0}(t) \delta y(0, t)
\end{aligned}
$$

where, $c, d_{s}$ and $d_{0}$ describe the damping coefficients of the pipe, vessel and ballast. Combining (5), (6) and (7), the total virtual work is deduced as:

$$
\delta W(t)=\delta W_{f}(t)+\delta W_{d}(t)+\delta W_{m}(t)
$$

Deduce the governing equation by substituting (2), (3), (8) into (1) and integrating by part

$$
\begin{aligned}
& \rho \ddot{y}(x, t)+E I y^{\prime \prime \prime \prime}(x, t)-T^{\prime}(x, t) y^{\prime}(x, t)-\lambda^{\prime}(x) y^{\prime 3}(x, t) \\
& -\left[T(x, t)+3 \lambda(x) y^{\prime 2}(x, t)\right] y^{\prime \prime}(x, t)=f(x, t)-c \dot{y}(x, t)(9)
\end{aligned}
$$

$\forall(x, t) \in(0, L) \times(0, \infty)$, and obtain the boundary conditions as:

$$
\begin{gathered}
y^{\prime}(0, t)=y^{\prime \prime}(L, t)=0 \\
M_{0} \ddot{y}(0, t)+E I y^{\prime \prime \prime}(0, t)+d_{0} \dot{y}(0, t)=u_{0}(t)+p_{0}(t)(11) \\
M_{s} \ddot{y}(L, t)-E I y^{\prime \prime \prime}(L, t)+d_{s} \dot{y}(L, t)+T(L, t) y^{\prime}(L, t) \\
+\lambda(L) y^{\prime 3}(L, t)=u_{1}(t)+p_{s}(t)(12)
\end{gathered}
$$

$\forall t \in(0, \infty)$.

Assumption 1: The unknown environmental disturbances $f(x, t), p_{s}(t)$ and $p_{0}(t)$ are assumed to be bounded and exist positive real constants $\bar{f}, \bar{p}_{s}$ and $\bar{p}_{0}$ to satisfy $|f(x, t)| \leq \bar{f}$, $\left|p_{s}(t)\right| \leq \bar{p}_{s}$ and $\left|p_{0}(t)\right| \leq \bar{p}_{0}$, where $\forall x \in(0, L)$ and $t \in[0, \infty)$.

Assumption 2: The initial tension $T_{0}(x)$ and elastic modulus $\lambda(x)$ of the pipe are assumed to be bounded and exist four positive real constants $T_{0 \min }, T_{0 \max }, \lambda_{\min }$, and $\lambda_{\max }$ to satisfy $T_{0} \in\left[T_{0 \min }, T_{0 \max }\right]$ and $\lambda \in\left[\lambda_{\min }, \lambda_{\max }\right], \forall x \in[0, L]$.

\section{CONTROL DESIGN}

The control objects are to design the adaptive vibration controls for the offshore OTEC system under the external disturbances, to estimate the unknown parameters and to maintain the boundary tension $T(L, t)$ restrain in the constrained space. The adaptive boundary controls with the barrier term depicted in Fig. 2 are designed to analyze the stability of the OTEC system.

The following robust adaptive boundary controls are pro-

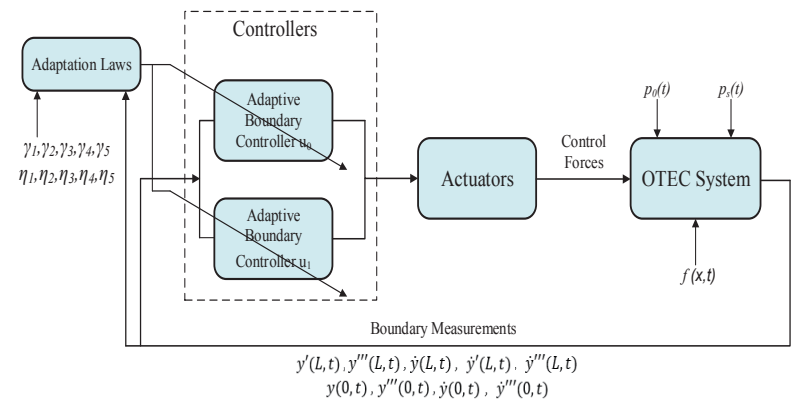

Fig. 2. The design scheme of robust adaptive boundary control laws

posed to deal with the undetermined parameters:

$$
\begin{aligned}
u_{0}(t)= & -\frac{k_{0}}{k_{4}} u_{a 0}(t)-\frac{u_{a 0}(t)\left[\frac{\widehat{M_{0}} y(0, t) \dot{y}(0, t)}{D_{0}^{2}-y^{2}(0, t)}+k_{s 0}\right]}{k_{4} \ln \frac{2 D_{0}^{2}}{D_{0}^{2}-y^{2}(0, t)}} \\
& -\operatorname{sgn}\left[u_{a 0}(t)\right] \bar{p}_{0}+d_{0} \dot{y}(0, t)-\frac{k_{5}}{k_{4}} \widehat{M_{0}}(t) \dot{y}^{\prime \prime \prime}(0, t) \\
& +\widehat{E I}(t) y^{\prime \prime \prime}(0, t) \\
u_{1}(t)=- & \frac{k_{1}}{k_{6}} u_{a 1}(t)-\frac{u_{a 1}(t)\left[k_{s 1}+\frac{\widehat{M_{s}}(t) y^{\prime}(L, t) \dot{y}^{\prime}(L, t)}{W_{0}^{2}-y^{\prime 2}(L, t)}\right]}{k_{6} \ln \frac{2 W_{0}^{2}}{W_{0}^{2}-y^{\prime 2}(L, t)}} \\
& -\operatorname{sgn}\left[u_{a 1}(t)\right] \bar{p}_{s}+d_{s} \dot{y}(L, t)-\frac{\widehat{M}_{s}(t)}{k_{6}}\left[k_{7} \dot{y}^{\prime}(L, t)\right. \\
& +\widehat{T_{0}}(L, t) y^{\prime}(L, t)+2 \widehat{\lambda}(L, t) y^{\prime 3}(L, t)-k_{2} \dot{y}^{\prime \prime \prime}(L, t) \\
& \left.-\widehat{E I}(t) y^{\prime \prime \prime}(L, t)+3 k_{3} y^{\prime 2}(L, t) \dot{y}^{\prime}(L, t)\right]
\end{aligned}
$$

where $k_{0}-k_{5}, k_{s 0}$ and $k_{s 1}$ are control gains. $D_{0}$ is the upper bound of $y(0, t)$ and $W_{0}$ represents the upper bound of $y^{\prime}(L, t)$ to restraint the boundary tension, and $\operatorname{sgn}(\cdot)$ denotes the signum function. $\widehat{T_{0}}(L, t), \widehat{\lambda}(L, t), \widehat{E I}(t), \widehat{M}_{s}(t), \widehat{M}_{0}(t)$ and wide $\hat{P}_{0}(t)$ and $\widehat{P}_{s}(t)$ represent the estimate values of $T_{0}(L), \lambda(L), E I, M_{s}, M_{0}, p_{0}(t)$ and $p_{s}(t)$ respectively. The estimate error $\widetilde{(\star)}$ is denoted as $(\star)=(\star)-(\star)$. The adaptive laws are designed as follows:

$$
\begin{aligned}
\dot{\widehat{T_{0}}}(L, t)= & -\beta \gamma_{1}^{-1} k_{6} u_{a 1}(t) y^{\prime}(L, t) \ln \frac{2 W_{0}^{2}}{W_{0}^{2}-y^{\prime 2}(L, t)} \\
& -\gamma_{1}^{-1} \eta_{1} \widehat{T_{0}}(L, t) \\
\dot{\hat{\lambda}}(L, t)= & -2 \beta \gamma_{2}^{-1} k_{6} u_{a 1}(t) y^{\prime 3}(L, t) \ln \frac{2 W_{0}^{2}}{W_{0}^{2}-y^{\prime 2}(L, t)} \\
& -\gamma_{2}^{-1} \eta_{2} \widehat{\lambda}(L, t) \\
\dot{\widehat{E I}}(t)= & \beta \gamma_{3}^{-1} k_{6} u_{a 1}(t) y^{\prime \prime \prime}(L, t) \ln \frac{2 W_{0}^{2}}{W_{0}^{2}-y^{\prime 2}(L, t)} \\
& -\beta k_{4} \gamma_{3}^{-1} u_{a 0}(t) y^{\prime \prime \prime}(0, t) \ln \frac{2 D_{0}^{2}}{D_{0}^{2}-y^{2}(0, t)} \\
& -\gamma_{3}^{-1} \eta_{3} \widehat{E I}(t) \\
\dot{\vec{M}_{s}}(t)= & \beta \gamma_{4}^{-1} u_{a 1}(t)\left[-k_{2} \dot{y}^{\prime \prime \prime}(L, t)+3 k_{3} y^{\prime 2}(L, t) \dot{y}^{\prime}(L, t)\right. \\
& \left.+k_{7} \dot{y}^{\prime}(L, t)\right] \ln \frac{2 W_{0}^{2}}{W_{0}^{2}-y^{\prime 2}(L, t)}-\gamma_{4}^{-1} \eta_{4} \widehat{M}_{s}(t)
\end{aligned}
$$




$$
\begin{aligned}
& +\beta \gamma_{4}^{-1} u_{a 1}^{2}(t) \frac{y^{\prime}(L, t) \dot{y}^{\prime}(L, t)}{W_{0}^{2}-y^{\prime 2}(L, t)} \\
\dot{\widehat{M}}_{0}(t)= & \beta k_{5} \gamma_{5}^{-1} u_{a 0}(t) \dot{y}^{\prime \prime \prime}(0, t) \ln \frac{2 D_{0}^{2}}{D_{0}^{2}-y^{2}(0, t)} \\
& +\beta \gamma_{5}^{-1} u_{a 0}^{2} \frac{y(0, t) \dot{y}(0, t)}{D_{0}^{2}-y^{2}(0, t)}-\gamma_{5}^{-1} \eta_{5} \widehat{M}_{0}(t)
\end{aligned}
$$

where $\gamma_{1}-\gamma_{5}$ are positive control parameters, and $\eta_{1}-\eta_{5}$ are small positive constants, and $u_{a 0}(t)$ and $u_{a 1}(t)$ are two auxiliary signals given as

$$
u_{a 0}(t)=k_{4} \dot{y}(0, t)+k_{5} y^{\prime \prime \prime}(0, t)
$$

$u_{a 1}(t)=-k_{2} y^{\prime \prime \prime}(L, t)+k_{3} y^{\prime 3}(L, t)+k_{6} \dot{y}(L, t)+k_{7} y^{\prime}(L, t)$

where $k_{6}$ and $k_{7}$ are positive control gains.

Construct the Lyapunov candidate function as

$$
H(t)=H_{1}(t)+H_{2}(t)+H_{3}(t)+H_{4}(t)
$$

where

$$
\begin{aligned}
H_{1}(t)= & \frac{\beta \rho}{2} \int_{0}^{L}[\dot{y}(x, t)]^{2} d x+\frac{\beta E I}{2} \int_{0}^{L}\left[y^{\prime \prime}(x, t)\right]^{2} d x \\
& +\frac{\beta}{2} \int_{0}^{L} T(x, t)\left[y^{\prime}(x, t)\right]^{2} d x \\
H_{2}(t)= & \frac{\beta}{2} M_{s} u_{a 1}^{2}(t) \ln \frac{2 W_{0}^{2}}{W_{0}^{2}-y^{\prime 2}(L, t)} \\
& +\frac{\beta}{2} M_{0} u_{a 0}^{2}(t) \ln \frac{2 D_{0}^{2}}{D_{0}^{2}-y^{2}(0, t)} \\
H_{3}(t)= & \alpha \rho \int_{0}^{L} x \dot{y}(x, t) y^{\prime}(x, t) d x \\
H_{4}(t)= & \frac{\gamma_{1}}{2} \widetilde{T}_{0}^{2}(L, t)+\frac{\gamma_{2}}{2} \widetilde{\lambda}^{2}(L, t)+\frac{\gamma_{3}}{2} \widetilde{E I}^{2}(t) \\
& +\frac{\gamma_{4}}{2} \widetilde{M}_{s}^{2}(t)+\frac{\gamma_{5}}{2} \widetilde{M}_{0}^{2}(t)
\end{aligned}
$$

where $\alpha$ and $\beta$ are two positive weights.

Lemma 1: The function (22) is proven to be bounded as:

$$
\begin{aligned}
0 & \leq \mu_{1}\left[H_{1}(t)+H_{2}(t)+H_{4}(t)\right] \leq H(t) \\
& \leq \mu_{2}\left[H_{1}(t)+H_{2}(t)+H_{4}(t)\right]
\end{aligned}
$$

where the two positive constants $\mu_{1}$ and $\mu_{2}$ are given as:

provided that:

$$
\begin{aligned}
& \mu_{1}=1-\frac{2 \alpha \rho L}{\beta \min \left(\rho, T_{0 \text { min }}\right)}>0 \\
& \mu_{2}=1+\frac{2 \alpha \rho L}{\beta \min \left(\rho, T_{0 \text { min }}\right)}>0
\end{aligned}
$$

$$
0 \leq \alpha \leq \frac{\beta \min \left(\rho, T_{0 \min }\right)}{2 \rho L}
$$

Proof: $H_{3}(t)$ can be yielded as

$$
\begin{aligned}
\left|H_{3}(t)\right| & \leq \alpha \rho L \int_{0}^{L}\left|\dot{y}(x, t) y^{\prime}(x, t)\right| d x \\
& \leq \alpha \rho L\left[\int_{0}^{L} \dot{y}^{2}(x, t) d x+\int_{0}^{L} y^{\prime 2}(x, t) d x\right]
\end{aligned}
$$

$$
\leq \alpha_{1} H_{1}(t)
$$

where $\alpha_{1}=\frac{2 \alpha \rho L}{\beta \min \left(\rho, T_{0 \min }\right)}$. Subsequently the above equation can be rewritten as

$$
-\alpha_{1} H_{1}(t) \leq H_{3}(t) \leq \alpha_{1} H_{1}(t)
$$

For the positive weight constant $\alpha$, the inequality $0 \leq \alpha \leq$ $\frac{\beta \min \left(\rho, T_{0 \min }\right)}{2 \rho L}$ holds, and we have

$$
\alpha_{2} H_{1}(t) \leq H_{1}(t)+H_{3}(t) \leq \alpha_{3} H_{1}(t)
$$

where

$$
\begin{aligned}
& \alpha_{2}=1-\frac{2 \alpha \rho L}{\beta \min \left(\rho, T_{0 \min }\right)}>0 \\
& \alpha_{3}=1+\frac{2 \alpha \rho L}{\beta \min \left(\rho, T_{0 \min }\right)}>1
\end{aligned}
$$

Therefore, we further have

$$
\begin{aligned}
0 & \leq \mu_{1}\left[H_{1}(t)+H_{2}(t)+H_{4}(t)\right] \leq H(t) \\
& \leq \mu_{2}\left[H_{1}(t)+H_{2}(t)+H_{4}(t)\right]
\end{aligned}
$$

where $\mu_{1}=\min \left(\alpha_{2}, 1\right)=\alpha_{2}$ and $\mu_{2}=\max \left(\alpha_{3}, 1\right)=\alpha_{3}$ are two positive constants. Subsequently, (27) is proven.

Lemma 2: The time derivative of the function (22) is bounded,

$$
\dot{H}(t) \leq-\mu H(t)+\varepsilon_{a}
$$

where $\mu$ and $\varepsilon_{a}$ are two positive constants.

Proof: The time derivative of (22) is yielded as

$$
\dot{H}(t)=\dot{H}_{1}(t)+\dot{H}_{2}(t)+\dot{H}_{3}(t)+\dot{H}_{4}(t)
$$

After substituting the governing equation (9), integrating by parts, and substituting auxiliary signals (21), (20), we obtain

$$
\begin{aligned}
\dot{H}_{1}(t)= & \frac{\beta E I}{2 k_{4} k_{5}}\left[u_{a 0}^{2}(t)-k_{4}^{2} \dot{y}^{2}(0, t)-k_{5}^{2} y^{\prime \prime \prime}(0, t)\right] \\
& +\frac{\beta T_{0}(L)}{2 k_{6} k_{7}}\left[u_{a 1}^{2}(t)-k_{6}^{2} \dot{y}^{2}(L, t)-k_{7}^{2} y^{\prime 2}(L, t)\right. \\
& \left.-k_{2}^{2} y^{\prime \prime \prime 2}(L, t)-k_{3}^{2} y^{\prime 6}(L, t)\right]-\frac{\beta k_{3} T_{0}(L)}{k_{6}} y^{\prime 4}(L, t) \\
& +\beta\left[\frac{k_{2} T_{0}(L)}{k_{7}}-E I\right] y^{\prime \prime \prime}(L, t) \dot{y}(L, t) \\
& +\beta\left[2 \lambda(L)-\frac{k_{3} T_{0}(L)}{k_{7}}\right] y^{\prime 3}(L, t) \dot{y}(L, t) \\
& +\beta \int_{0}^{L} f(x, t) \dot{y}(x, t) d x-\beta \int_{0}^{L} c \dot{y}^{2}(x, t) d x \\
& +\frac{\beta k_{2} k_{3} T_{0}(L)}{k_{6} k_{7}} y^{\prime \prime \prime}(L, t) y^{\prime 3}(L, t) \\
& +\frac{\beta k_{2} T_{0}(L)}{k_{6}} y^{\prime}(L, t) y^{\prime \prime \prime}(L, t)
\end{aligned}
$$

Substituting the boundary conditions (11), (12), then 
proposing the controllers (13) and (14), $\dot{H}_{2}(t)$ is given by

$$
\begin{aligned}
\dot{H}_{2}(t)= & -k_{1} \beta u_{a 1}^{2}(t) \ln \frac{2 W_{0}^{2}}{W_{0}^{2}-y^{\prime 2}(L, t)}-k_{s 1} \beta u_{a 1}^{2}(t) \\
& -k_{0} \beta u_{a 0}^{2}(t) \ln \frac{2 D_{0}^{2}}{D_{0}^{2}-y^{2}(0, t)}-k_{s 0} \beta u_{a 0}^{2}(t) \\
& +\beta u_{a 1}(t) \ln \frac{2 W_{0}^{2}}{W_{0}^{2}-y^{\prime 2}(L, t)}\left\{k_{6} \widetilde{T_{0}}(L, t) y^{\prime}(L, t)\right. \\
& +2 k_{6} \widetilde{\lambda}(L, t) y^{\prime 3}(L, t)-k_{6} \widetilde{E I}(t) y^{\prime \prime \prime}(L, t) \\
& -\widetilde{M}_{s}(t)\left[k_{7} \dot{y}^{\prime}(L, t)+3 k_{3} y^{\prime 2}(L, t) \dot{y}^{\prime}(L, t)\right. \\
& \left.\left.-k_{2} \dot{y}^{\prime \prime \prime}(L, t)\right]\right\}-\frac{\beta u_{a 1}^{2} \widetilde{M}_{s}(t) y^{\prime}(L, t) \dot{y}^{\prime}(L, t)}{W_{0}^{2}-y^{\prime 2}(L, t)} \\
& -\frac{\beta u_{a 0}^{2} \widetilde{M}_{0}(t) y(0, t) \dot{y}(0, t)}{D_{0}^{2}-y^{2}(0, t)} \\
+ & \beta u_{a 0}(t) \ln \frac{2 D_{0}^{2}}{D_{0}^{2}-y^{2}(0, t)}\left[k_{4} \widetilde{E I}(t) y^{\prime \prime \prime}(0, t)\right. \\
- & \left.k_{5} \widetilde{M}_{0}(t) \dot{y}^{\prime \prime \prime}(0, t)\right]
\end{aligned}
$$

Substituting the governing equation (9), integrating by parts, the third term of (35) is shown as

$$
\begin{aligned}
\dot{H}_{3}(t) \leq & -\frac{\alpha}{2} \int_{0}^{L}\left[T_{0}(L)-x T_{0}^{\prime}(x)\right] y^{\prime 2}(x, t) d x \\
& +\frac{\alpha L}{\delta_{1}} \int_{0}^{L} f^{2}(x, t) d x+\frac{3 \alpha}{2} L \lambda(L) y^{\prime 4}(L, t) \\
& +\frac{\alpha \rho L}{2} \dot{y}^{2}(L, t)-\frac{\alpha \rho}{2} \int_{0}^{L} \dot{y}^{2}(x, t) d x \\
& +\alpha L\left(\delta_{1}+c \delta_{2}\right) \int_{0}^{L} y^{\prime 2}(x, t) d x \\
& -\frac{\alpha}{2} \int_{0}^{L}\left[3 \lambda(x)-x \lambda^{\prime}(x)\right] y^{\prime 4}(x, t) d x \\
& +\frac{\alpha c L}{\delta_{2}} \int_{0}^{L} \dot{y}^{2}(x, t) d x+\frac{\alpha}{2} L T_{0}(L) y^{\prime 2}(L, t) \\
& -\alpha E I L y^{\prime}(L, t) y^{\prime \prime \prime}(L, t) \\
& -\frac{3 \alpha E I L}{2} \int_{0}^{L} y^{\prime \prime 2}(x, t) d x
\end{aligned}
$$

where $\delta_{1}$ and $\delta_{2}$ are positive constants.

The derivative of $H_{4}(t)$ is obtained as

$$
\begin{aligned}
\dot{H}_{4}(t)= & \gamma_{1} \widetilde{T_{0}}(L, t) \dot{\widetilde{T_{0}}}(L, t)+\gamma_{2} \widetilde{\lambda}(L, t) \dot{\widetilde{\lambda}}(L, t) \\
& +\gamma_{3} \widetilde{E I}(t) \dot{\vec{E}}(t)+\gamma_{4} \widetilde{M}_{s}(t) \dot{\widetilde{M}}_{s}(t) \\
& +\gamma_{5} \widetilde{M}_{0}(t) \dot{\dot{\widetilde{M}}_{0}}(t)
\end{aligned}
$$

After substituting (36) - (39) and adaptive laws (15) - (19) into (35), we acquire that

$$
\begin{aligned}
\dot{H}(t) \leq & -\beta\left[k_{s 1}-\frac{T_{0}(L)}{2 k_{6} k_{7}}+k_{1} \ln \frac{2 W_{0}^{2}}{W_{0}^{2}-y^{\prime 2}(L, t)}\right] u_{a 1}^{2}(t) \\
& -\beta\left[k_{s 0}-\frac{E I}{2 k_{4} k_{5}}+k_{0} \ln \frac{2 D_{0}^{2}}{D_{0}^{2}-y^{2}(0, t)}\right] u_{a 0}^{2}(t) \\
& -\beta \theta_{1} \dot{y}^{2}(L, t)-\int_{0}^{L} \sigma_{1} \dot{y}^{2}(x, t) d x-\theta_{2} y^{\prime 2}(L, t) \\
& -\int_{0}^{L} \sigma_{2} y^{\prime 4}(x, t) d x-\int_{0}^{L} \sigma_{3} y^{\prime 2}(x, t) d x \\
& -\theta_{3} y^{\prime \prime \prime}(L, t)-\beta \theta_{4} y^{\prime 6}(L, t)-\int_{0}^{L} \sigma_{4} y^{\prime \prime 2}(x, t) d x \\
& -\frac{\eta_{1}}{2}{\widetilde{T_{0}}}^{2}(L, t)-\theta_{5} y^{\prime 4}(L, t)-\frac{\beta k_{4} E I}{2 k_{5}} \dot{y}^{2}(0, t) \\
& -\frac{\beta k_{5} E I}{2 k_{4}} y^{\prime \prime \prime 2}(0, t)-\frac{\eta_{2}}{2} \widetilde{\lambda}^{2}(L, t)-\frac{\eta_{3}}{2} \widetilde{E I}^{2}(t) \\
& -\frac{\eta_{4}}{2} \widetilde{M}_{s}^{2}(t)-\frac{\eta_{5}}{2} \widetilde{M}_{0}^{2}(t)+\varepsilon_{a}
\end{aligned}
$$

where $\delta_{3} \sim \delta_{7}$ are positive constants and relative parameters are chosen to satisfy:

$$
\begin{aligned}
\sigma_{1} & =\frac{\alpha \rho}{2}-\frac{\alpha c L}{\delta_{2}}+\beta c-\frac{\beta}{\delta_{7}} \geq 0 \\
\sigma_{2} & =\frac{\alpha}{2}\left[3 \lambda(x)-x \lambda^{\prime}(x)\right] \geq 0 \\
\sigma_{3} & =\frac{\alpha}{2}\left[T_{0}(L)-\frac{\alpha}{2} x T_{0}^{\prime}(x)\right]-\alpha L\left(\delta_{1}+c \delta_{2}\right) \geq 0 \\
\sigma_{4} & =\frac{3 \alpha E I L}{2} \geq 0
\end{aligned}
$$

and

$$
\begin{aligned}
& k_{s 1}-\frac{T_{0}(L)}{2} \geq 0 \\
& k_{s 0}-\frac{E I}{2 k_{4} k_{5}} \geq 0
\end{aligned}
$$

and

$$
\begin{aligned}
\theta_{1}= & \frac{k_{6} T_{0}(L)}{2 k_{7}}-\frac{\left|k_{2} T_{0}(L)-k_{7} E I\right|}{k_{7} \delta_{3}}-\frac{\left|2 k_{7} \lambda(L)-k_{3} T_{0}(L)\right|}{k_{7} \delta_{4}} \\
& -\frac{\alpha \rho L}{2} \geq 0 \\
\theta_{2}= & \beta \frac{k_{7} T_{0}(L)}{2 k_{6}}-\left|\frac{\beta k_{2} T_{0}(L)}{k_{6}}-\alpha E I L\right| \delta_{5} \\
& -\frac{\alpha L}{2} T_{0}(L) \geq 0 \\
\theta_{3}= & \frac{k_{2}^{2} \beta T_{0}(L)}{2 k_{6} k_{7}}-\beta\left|\frac{k_{2} T_{0}(L)}{k_{7}}-E I\right| \delta_{3}-\frac{\beta k_{2} k_{3} T_{0}(L) \delta_{6}}{k_{6} k_{7}} \\
& -\frac{\left|\beta k_{2} T_{0}(L)-\alpha k_{6} E I L\right|}{k_{6} \delta_{5}} \geq 0 \\
\theta_{4}= & \frac{T_{0}(L) k_{3}^{2}}{2 k_{6} k_{7}}-\left|2 \lambda(L)-\frac{k_{3} T_{0}(L)}{k_{7}}\right| \delta_{4}-\frac{T_{0}(L) k_{2} k_{3}}{k_{6} k_{7} \delta_{6}} \geq 0
\end{aligned}
$$

$\theta_{5}=\frac{\beta k_{3} T_{0}(L)}{k_{6}}-\frac{3 \alpha}{2} L \lambda(L) \geq 0$ 
and

$$
\begin{aligned}
& \mu_{3}= \min \left(\frac{2 \sigma_{1}}{\beta \rho}, \frac{2 \sigma_{2}}{\beta \lambda_{\max }}, \frac{2 \sigma_{3}}{\beta T_{0 \max }}, \frac{2 \sigma_{4}}{\beta E I}, \frac{2 k_{1}}{M_{s}},\right. \\
&\left.\frac{2 k_{0}}{M_{0}}, \frac{\eta_{i}}{\gamma_{i}}(i=1, \cdots, 5),\right) \\
& \varepsilon_{a}=\left(\beta \delta_{7}+\frac{\alpha L}{\delta_{1}}\right) L \bar{f}^{2}+\frac{\eta_{1}}{2} T_{0}^{2}(L)+\frac{\eta_{2}}{2} \lambda^{2}(L) \\
&+\frac{\eta_{3}}{2} E I^{2}+\frac{\eta_{4}}{2} M_{s}^{2}+\frac{\eta_{5}}{2} M_{0}^{2}
\end{aligned}
$$

Then, supplying (27) leads to:

$$
\dot{H}(t) \leq-\mu H(t)+\varepsilon_{a}
$$

where $\mu=\mu_{3} / \mu_{2 a}>0$ and $\varepsilon_{a}>0$.

When (27) and (34) are proven, the following theorem of the offshore OTEC system is analyzed.

Theorem 1: For the OTEC system (9)-(12), with the bounded initial conditions and boundary controls (13)-(19), the following conclusions can be obtained:

1) The states of the system is convergence and the stability of the system is obtained;

2) These estimation errors of the parameters are uniformly bounded;

3) When the initial states $y(0,0)$ and $y^{\prime}(L, 0)$ do not violate the constraints $D_{0}$ and $W_{0}$, the output signals $y(0, t)$ and $y^{\prime}(L, t)$ can be constrained as $|y(0, t)|<D_{0}$ and $\left|y^{\prime}(L, t)\right|<W_{0}$. Further, the constrained distributed tension cannot be violated.

Proof: Multiplying (34) by $e^{\mu t}$ leads to:

$$
\begin{aligned}
\frac{\partial}{\partial t}\left(H(t) e^{\mu t}\right) & \leq \varepsilon_{a} e^{\mu t} \\
H(t) & \leq\left(H(0)-\frac{\varepsilon_{a}}{\mu}\right) e^{-\mu t}+\frac{\varepsilon_{a}}{\mu} \in \mathcal{L}_{\infty}
\end{aligned}
$$

From the above inequality, we can obtain that $H(t)$ is bounded. Further, according to Assumption 2, the inequality is obtained

$$
\begin{aligned}
& \frac{\beta T_{0 \min }}{2 L}[y(x, t)-y(0, t)]^{2} \leq \frac{\beta}{2} \int_{0}^{L} T_{0}(x) y^{\prime 2}(x, t) d x \\
& \leq H_{1}(t)+H_{2}(t) \leq \frac{H(t)}{\mu_{1 a}}
\end{aligned}
$$

Finally, we have:

$$
|y(x, t)| \leq \sqrt{\frac{2 L}{\beta \mu_{1 a} T_{0 \min }}\left[H(0) e^{-\mu t}+\frac{\varepsilon_{a}}{\mu}\right]}+D_{0}
$$

with the same method, we have that estimation errors of the parameters are uniformly bounded and given as $|\tilde{*}(t)| \leq \sqrt{\frac{2}{\mu_{1 a} \gamma_{i}}\left[H(0) e^{-\mu t}+\frac{\varepsilon_{a}}{\mu}\right]}$, where, $*=$ $\left(T_{0}(L), \lambda(L), E I, M_{s}, M_{0}\right)$ and $i=(1,2,3,4,5)$, respectively.

$$
\lim _{t \rightarrow \infty}|y(x, t)| \leq \sqrt{\frac{2 \varepsilon_{a} L}{\beta T_{0 \min } \mu_{1 a} \mu}}+D_{0}
$$

Remark 1: From the definition of the Lyapunov function and proof of the Theorem 1, it is clear that $H_{2}(t)$ is positive and bounded. Therefore, we can deduce that the signals $y(0, t)$ and $y^{\prime}(L, t)$ can not be reach the desired limitations $D_{0}$ and $W_{0}$. When the initial values $y(0,0)$ and $y^{\prime}(L, 0)$ do not also violate the limitations, we conclude that the constraint problem for the output signals $y(0, t)$ and $y^{\prime}(L, t)$ can be solved as $|y(0, t)|<D_{0}$ and $\left|y^{\prime}(L, t)\right|<W_{0}$. From the expression of $T(x, t)(4)$, where the functions $T_{0}(x)$ and $\lambda(x)$ are bounded and known, it is easy to know that the tension of the flexible pipe is related to the state $y^{\prime}(x, t)$. In the other words, boundary tension $T(L, t)$ will not violate the given top tension constraint due to the determined parameter $T_{0}(L)$ and $\lambda(L)$ when the boundary state $y^{\prime}(L, t)$ is proven to satisfy the output constraint.

Remark 2: The designed adaptive boundary control laws and the disturbance observers are just designed with the measured boundary signals and their one-order-differential time derivatives, in order to weaken effects of the possible measure noise. These measurable signals are obtained by three class of sensors mounted at the boundaries of the pipe, namely, laser displacement sensor for signals $y(0, t)$ and $y(L, t)$, inclinometer for signals $y^{\prime}(0, t)$ and $y^{\prime}(L, t)$, and shear force sensor for signals $y^{\prime \prime \prime}(0, t)$ and $y^{\prime \prime \prime}(L, t)$. In addition, their one-order-differential time derivatives $\dot{y}(0, t)$, $\dot{y}(L, t), \dot{y}^{\prime}(0, t), \dot{y}^{\prime}(L, t), \dot{y}^{\prime \prime \prime}(0, t)$ and $\dot{y}^{\prime \prime \prime}(L, t)$ are calculated using a backward difference algorithm. From the viewpoint of implementation, these designed control strategies are realisable because of the install position and the number of sensors actuators for the OTEC system.

\section{Simulation}

To test the designed control method, numerical simulations are given out. The governing differential equation as well as boundary conditions are all discretized via finite difference method (FDM) and the transverse displacement $y(x, t)$ is calculated by computer. The control effects are presented through graphics.

\section{A. The definition of the disturbances}

The distributed disturbance $f(x, t)$ is described and consisted of mean and oscillating drags [59]. It is noted that the latter is $20 \%$ of the former term [60].

$$
f(x, t)=\frac{1}{2} \rho_{s} C_{D}(x, t) U^{2}(x, t) D+A_{D} \cos \left(4 \pi f_{v} t+\theta\right)
$$

where $\rho_{s}$ denotes the sea water density, the outer diameter $D$ and the drag coefficient $C_{D}$ are the parameters of the mean drag force. The phase angle $\theta$ and the amplitude $A_{D}$ are used to describe the oscillating drag. The vortex shedding frequency is given as:

$$
f_{v}=\frac{S_{t} U(x, t)}{D}
$$

where $S_{t}$ denotes the Strouhal number. The relationship between the distributed current $U(x, t)$ alone the pipe and the ocean face current $U(t)$ is given:

$$
\begin{aligned}
U(x, t) & =\frac{x}{1000} U(t) \\
U(t) & =\bar{U}+\sum_{i=1}^{4} U_{i}^{\prime} \sin \left(\omega_{i} t\right),
\end{aligned}
$$


In our simulation, we set the relating parameters as [61]:

TABLE I

PARAMETERS OF THE DISTRIBUTED DISTURBANCE

\begin{tabular}{|c|c|c|c|}
\hline Parameter & Value & Parameter & Value \\
\hline$U_{1}^{\prime}$ & $0.8 \mathrm{~m} / \mathrm{s}$ & $\omega_{3}$ & $2.946 \mathrm{rad} / \mathrm{s}$ \\
$U_{2}^{\prime}$ & $1.0 \mathrm{~m} / \mathrm{s}$ & $\omega_{4}$ & $4.282 \mathrm{rad} / \mathrm{s}$ \\
$U_{3}^{\prime}$ & $1.0 \mathrm{~m} / \mathrm{s}$ & $D$ & $0.15 \mathrm{~m}$ \\
$U_{4}^{\prime}$ & $1.0 \mathrm{~m} / \mathrm{s}$ & $\rho_{s}$ & $1024.0 \mathrm{~kg} / \mathrm{m}^{3}$ \\
$\bar{U}$ & $0.5 \mathrm{~m} / \mathrm{s}$ & $S_{t}$ & 0.2 \\
$\theta$ & $0 \mathrm{rad}$ & $A_{D}$ & 1.0 \\
$\omega_{1}$ & $0.867 \mathrm{rad} / \mathrm{s}$ & $C_{D}$ & 1.0 \\
$\omega_{2}$ & $1.827 \mathrm{rad} / \mathrm{s}$ & & \\
\hline
\end{tabular}

TABLE II

PARAMETERS OF THE OFFSHORE OTEC SYSTEM

\begin{tabular}{|c|c|c|c|}
\hline Parameter & Value & Parameter & Value \\
\hline$L$ & $1000 \mathrm{~m}$ & $\rho$ & $500 \mathrm{~kg} / \mathrm{m}$ \\
$E I$ & $2.5 \times 10^{4} \mathrm{~N} \cdot \mathrm{m}^{2}$ & $T_{0}(x)$ & $(x+1000) \times 10^{4} \mathrm{~N}$ \\
$\lambda(x)$ & $(x+1000) \times 10^{2}$ & $M_{s}$ & $9.0 \times 10^{2} \mathrm{~kg}$ \\
$M_{0}$ & $9.5 \times 10^{6} \mathrm{~kg}$ & $c$ & $2 \mathrm{~N} \cdot \mathrm{s} / \mathrm{m}^{2}$ \\
$d_{s}$ & $2.0 \times 10^{3} \mathrm{~N} \cdot \mathrm{s} / \mathrm{m}^{2}$ & $d_{0}$ & $9.0 \times 10^{2} \mathrm{~N} \cdot \mathrm{s} / \mathrm{m}^{2}$ \\
\hline
\end{tabular}

The environmental disturbance on the ballast caused by ocean wave is set without loss of generality as:

$$
p_{s}(t)=1.024 \times 10^{3} \times U(t)^{2} ;
$$

The environmental disturbance on the vessel [62], [63] is set without loss of generality as:

$$
p_{0}(t)=[0.8 \sin (0.2 t)+0.5 \sin (0.5 t)+0.2 \sin (0.7 t)] \times 10^{4} .
$$

\section{B. Control performance}

The initial condition is set as: $y(x, 0)=\frac{x}{L}$ and $\dot{y}(x, 0)=0$, where $x \in[0, L]$.

Considering the dynamics of the offshore OTEC system without control, according to Figs. 3 - 4, it can be observed that due to the disturbances $f(x, t), p_{s}(t)$ and $p_{0}(t)$, the OTEC system generates quite large vibration and the displacement of the vessel will increase continuously with the increased time. The vibration of the system's body is quite harmful during mechanical operation and effective control method should be designed to stabilize the vibration.

As a typical control strategy, PD control is proposed to reduce the vibrations of the flexible system. For this simple control, proportional and differential parameters are chosen to construct the simply control $u_{0}(t)=-100 y(0, t)-7000 \dot{y}(0, t)$ and $u_{1}(t)=-100 y(L, t)-8000 \dot{y}(L, t)$ and to adjust the dynamical characteristic of the closed-loop system. Under the constructed PD control, some snapshots are obtained to illustrate the positions of the flexible pipe in Fig. 6. Specifically, Figs. 7 and 8 depict the displacements of the ballast and the vessel on the ocean surface.

By disposing the two controllers on each boundary of the system, we have proposed the effective adaptive boundary control to reduce the vibration and maintain the output states within the constraint spaces, whose effectiveness can be seen in Fig. 9. The control gains are given as $k_{0}=1.0 \times 10^{8}$, $k_{1}=1.0 \times 10^{2}, k_{2}=0.75, k_{3}=100, k_{4}=100, k_{5}=0.001$, $k_{6}=0.001, k_{7}=0.5, k_{s 0}=5 \times 10^{8}$ and $k_{s 1}=1.5 \times 10^{7}$.
The constraints are set as $W_{0}=2.5, T_{c}=2.125 \times 10^{7}$ $\mathrm{N}$ and $D_{0}=0.15 \mathrm{~m}$. The parameters of adaptation laws are set as $\beta=1, \gamma_{i}=0.002, \quad(i=1, \cdots, 5)$ and $\eta_{1}=0.02, \quad(i=1, \cdots, 5)$. These parameters are selected mainly by trails on simulations and we have proved that all of them satisfy the inequations (41) - (47).

In terms of Fig. 9, the vibration has been suppressed into a small neighborhood of zero, whose upper bound is about $2 \mathrm{~m}$. According to Fig. 10, the top movement is suppressed as a minor periodic trajectory from $0 \mathrm{~m}$ to $1.2 \times 10^{-5} \mathrm{~m}$ with respect to time. The bottom movement is diminished into a vibration around $2 \mathrm{~m}$ subsequently, which can be seen in Fig. 11. From Fig. 10 and Fig. 12, both the two controllers can ensure their constraint projects, i.e., $|y(0, t)|<D_{0}$ and $\left|y^{\prime}(L, t)\right|<W_{0}$. With the definition of $T(L, t)$ and $W_{0}$, we further have $|T(L, t)|<T_{c}$ in Fig. 13. The control outputs of the robust adaptive controllers are shown in Fig. 14.

Differ from the situation without any control, it is seen that there exist control effect on the proposed control strategies, namely, the displacement of the flexible pipe is reduced and the vessel in the ocean surface is kept around the origin position. Comparing with the control performances of these two control methods, the developed vibration control (13)-(19) is better effective for vibration suppression of the OTEC system.

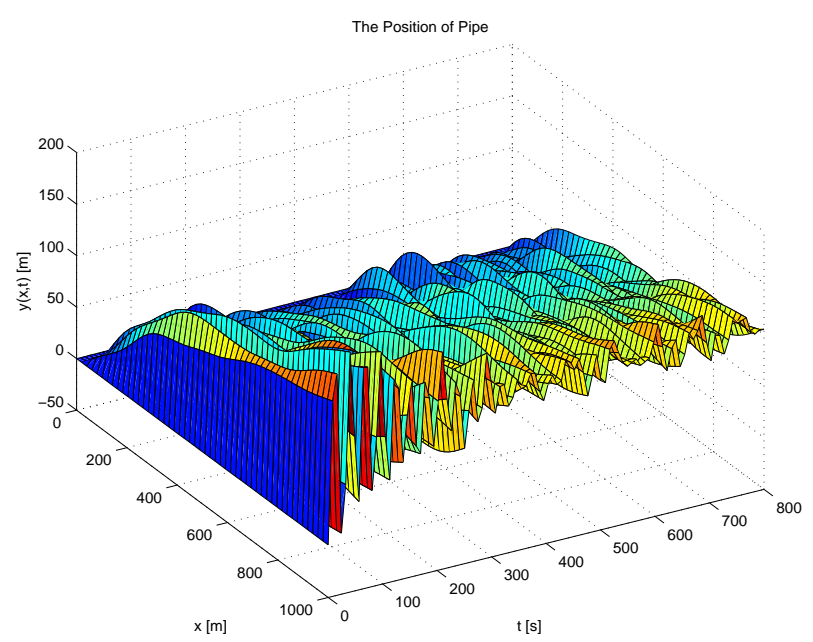

Fig. 3. Displacement of the OTEC system without control

\section{CONClusion}

An offshore OTEC system composed with a vessel, a long cold water pipe and a ballast has been consideration. The cooperative control method is constructed based on the hybrid PDE-ODEs system model to suppress the transverse vibrations and handle the constraint problems of the output signals and boundary tension. The stability of the control method has been certified via Lyapunov's direct method. Numerical experiments have been made to verify the theoretically proven adaptive controllers finally. In this paper, we research the vibration control of the OTEC system in one-dimensional space in order to deal with the constraint problem and uncertain parameter problem. However, the vibrations of the flexible pipe are three dimensional in the actual ocean environment. There exists 


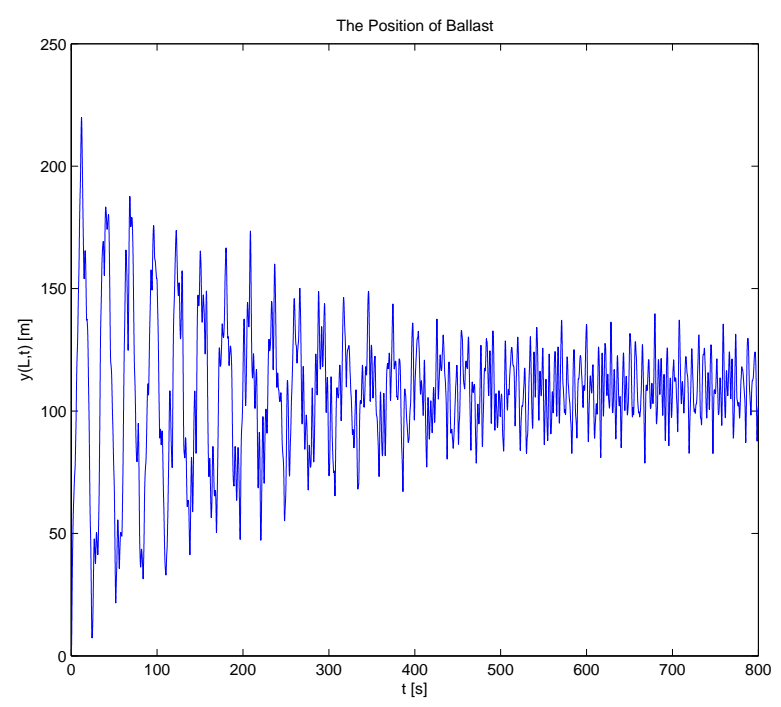

Fig. 4. Displacement of the ballast without the proposed control

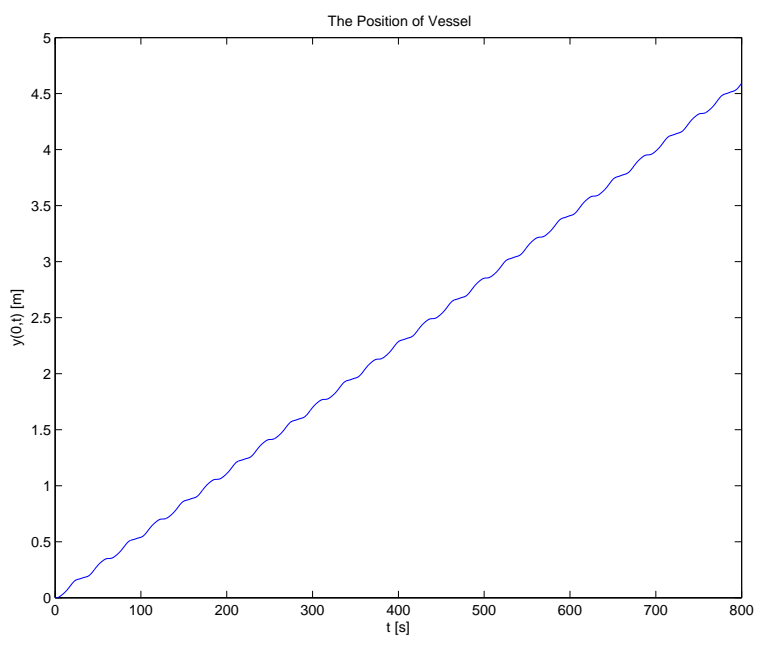

Fig. 5. Displacement of the vessel without the proposed control

strong coupling between the different directions and it is more difficult to design the effective vibration control to deal with the vibration suppression problem, constraint problem and the uncertain problem simultaneously for the OTEC system in the two or three dimensional space. It is a good topic to research this system in two or three dimensional spaces. In addition, taking the input constraints into consideration in this system is also a challenging work.

\section{ACKNOWLEDGMENTS}

The authors would like to sincerely thank the Editor-InChief, the Associate Editor and the anonymous reviewers for their constructive comments which helped improve the quality and presentation of this paper.

\section{REFERENCES}

[1] S. Zhan and G. Li, "Linear optimal noncausal control of wave energy converters," IEEE Transactions on Control Systems Technology, 2018, in press, DOI: 10.1109/TCST.2018.2812740.

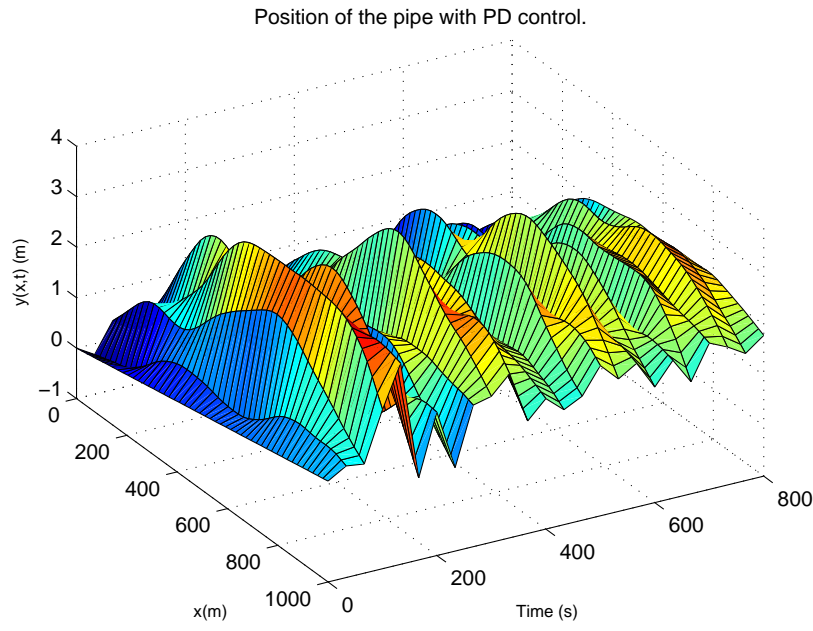

Fig. 6. Displacement of the OTEC system with PD control

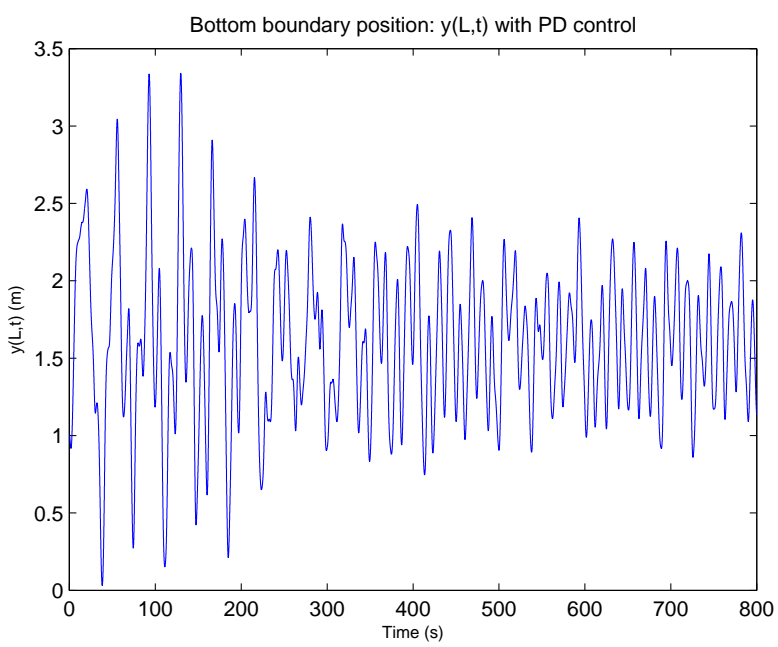

Fig. 7. Displacement of the ballast with PD control

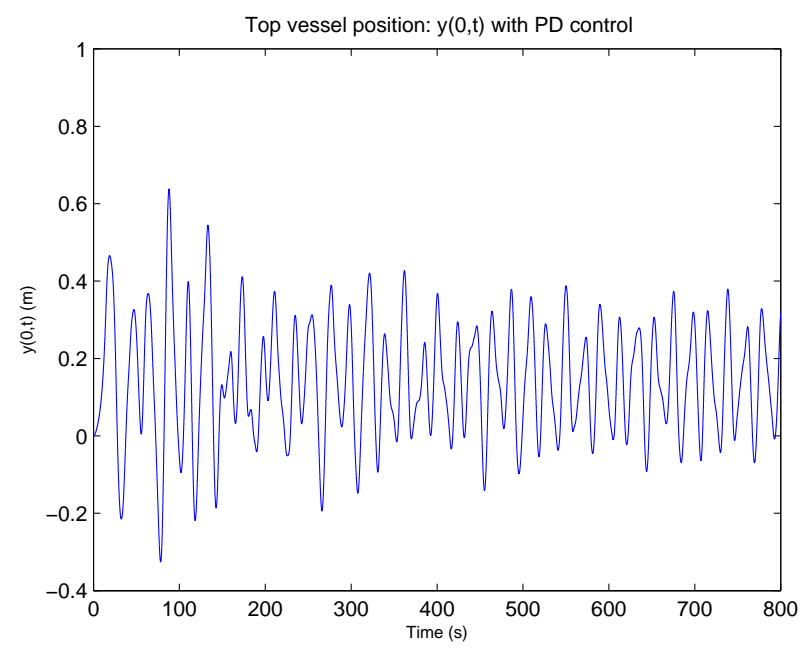

Fig. 8. Displacement of the vessel with PD control

[2] R.-H. Yeh, T.-Z. Su, and M.-S. Yang, "Maximum output of an OTEC power plant," Ocean Engineering, vol. 32, no. 5-6, pp. 685-700, 2005. 


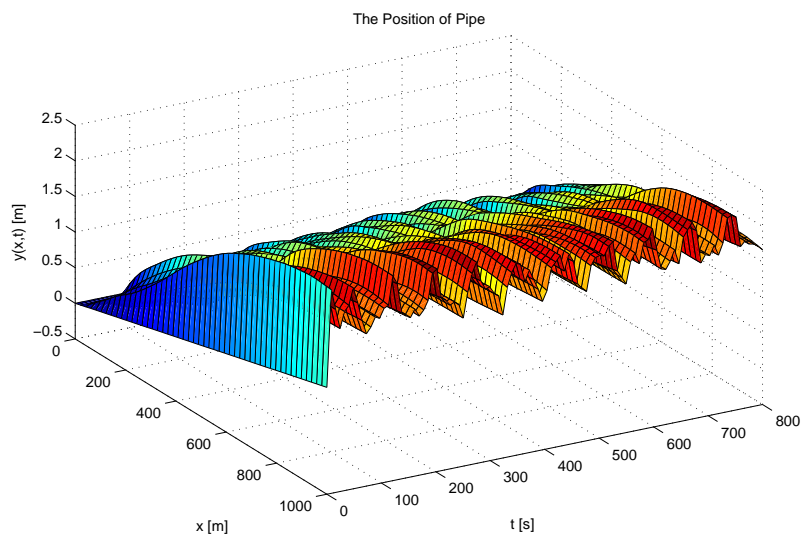

Fig. 9. Displacement of the OTEC system with the proposed control

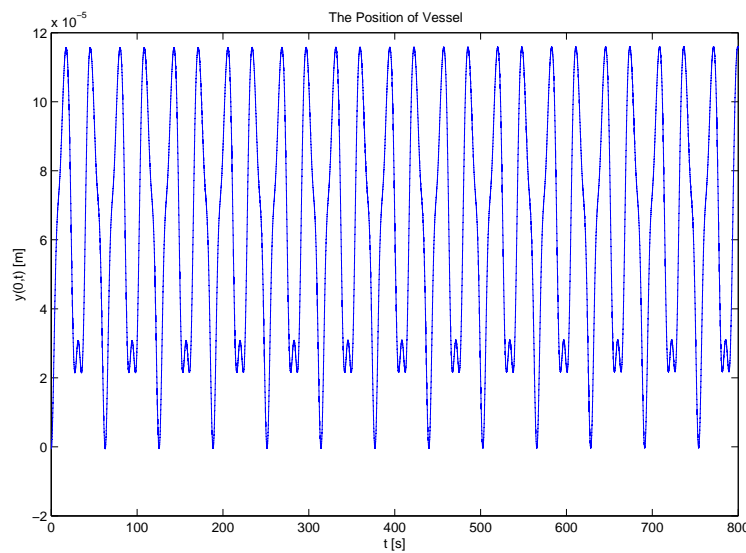

Fig. 10. Displacement of the vessel with the proposed control

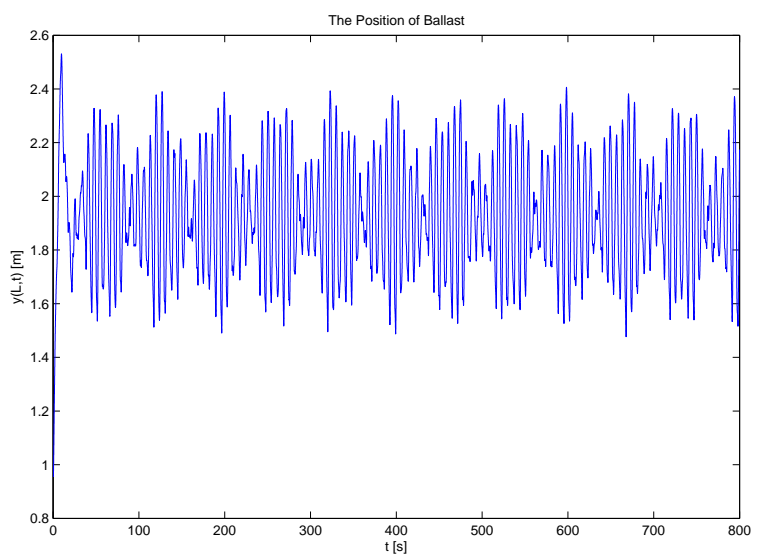

Fig. 11. Displacement of the ballast with the proposed control

[3] A. Gill and C. Black, "Status of hawaii's ocean energy projects and permitting regime," OCEANS 2011, pp. 1-8, 2011.

[4] J. Vadus and B. Taylor, "OTEC cold-water pipe research," IEEE Journal of Oceanic Engineering, vol. 10, no. 2, pp. 114-122, 1985.

[5] B. F. Tchanche, M. Ptrissans, and G. Papadakis, "Heat resources and organic rankine cycle machines," Renewable and Sustainable Energy Reviews, vol. 39, pp. 1185-1199, 2014.

[6] M. Faizal and M. R. Ahmed, "Experimental studies on a closed cycle demonstration OTEC plant working on small temperature difference," Renewable Energy, vol. 51, pp. 234-240, 2013.

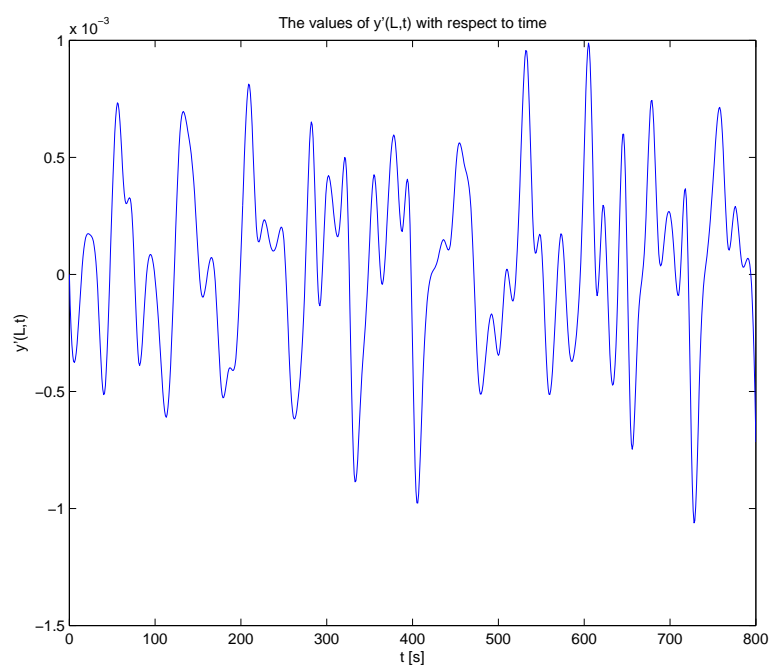

Fig. 12. The values of $y^{\prime}(L, t)$ with respect to $t$

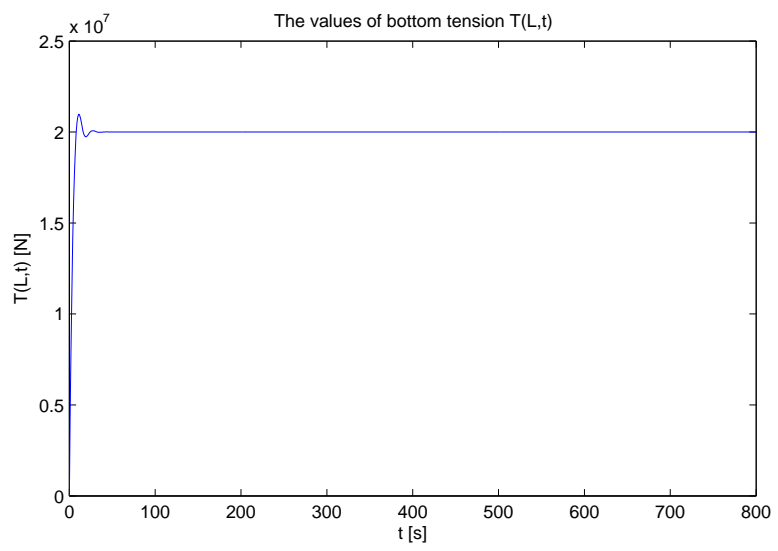

Fig. 13. The values of bottom tension $T(L, t)$ with respect to $t$
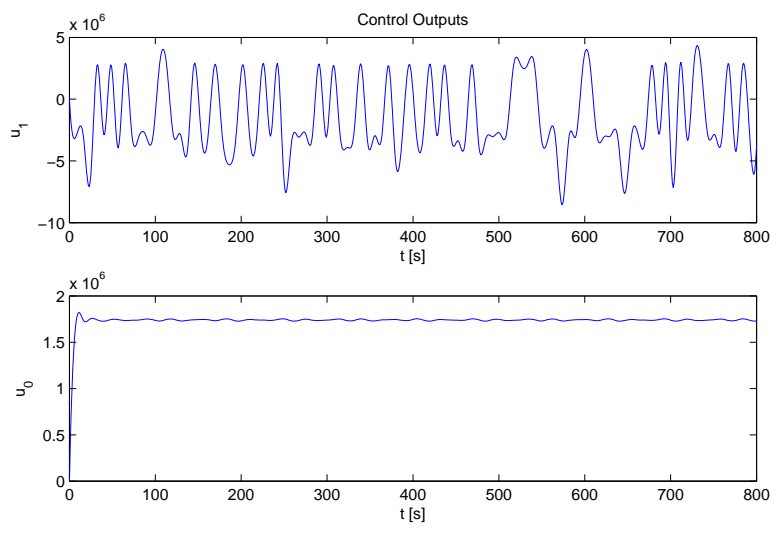

Fig. 14. The control outputs of $u_{0}$ and $u_{1}$

[7] G. C. Nihous, "Synchronous power output fluctuations for an experimental open-cycle OTEC plant," IEEE Transactions on Energy Conversion, vol. 12, no. 4, pp. 282-288, 1997.

[8] K. Rajagopalan and G. C. Nihous, "Estimates of global ocean thermal energy conversion (OTEC) resources using an ocean general circulation model," Renewable Energy, vol. 50, pp. 532-540, 2013.

[9] G. C. Nihous, "An estimate of atlantic ocean thermal energy conversion (OTEC) resources," Ocean Engineering, pp. 2210-2221, 2007. 
[10] P. J. Straatman and W. G. van Sark, "A new hybrid ocean thermal energy conversioncoffshore solar pond (OTEC - OSP) design: A cost optimization approach," Solar Energy, vol. 82, no. 6, pp. 520-527, 2007.

[11] H. Yuan, N. Mei, S. Hu, L. Wang, and S. Yang, "Experimental investigation on an ammonia-water based ocean thermal energy conversion system," Applied Thermal Engineering, vol. 61, no. 2, pp. 327-333, 2013.

[12] L. Wang and C.-C. Huang, "Dynamic stability analysis of a gridconnected solar-concentrated ocean thermal energy conversion system," IEEE Transactions on Sustainable Energy, vol. 1, no. 1, pp. 10-18, 2010.

[13] S. Goto, S. Kondoh, T. S. Yasuyuki Ikegam, and M. Nakamura, "Controller design for OTEC experimental pilot plant based on nonlinear separation control," SICE 2004 Annual Conference, vol. 1, pp. 879-884, 2004.

[14] S. Goto, D. Iseri, Y. Matsuda, T. Sugi, T. Morisaki, and Y. Ikegami, "Simulation model of evaporator and separator in OTEC plant using uehara cycle for liquid level control," SICE 2012 Annual Conference, pp. 2088-2093, 2012.

[15] A. Bhatia, S. Agarwal, and S. Khurana, "Optimal use of waste heat of condenser of thermal power plant," The 2nd International Conference on Computer and Automation Engineering (ICCAE), vol. 5, pp. 330-333, 2010.

[16] N. J. Kima, K. C. Ngb, and W. Chun, "Using the condenser effluent from a nuclear power plant for ocean thermal energy conversion (OTEC)," International Communications in Heat and Mass Transfer, vol. 36, no. 10, pp. 1008-1013, 2009.

[17] A. Smyshlyaev, B.-Z. Guo, and M. Krstic, "Arbitrary Decay Rate for Euler-Bernoulli Beam by Backstepping Boundary Feedback," IEEE Transactions on Automatic Control, vol. 54, no. 5, p. 1135, 2009.

[18] K. D. Do, "Modeling and boundary control of translational and rotational motions of nonlinear slender beams in three-dimensional space," Journal of Sound and Vibration, vol. 389, pp. 1-23, 2017.

[19] T. Endo, M. Sasaki, F. Matsuno, and Y. Jia, "Contact-force control of a flexible timoshenko arm in rigid/soft environment," IEEE Transactions on Automatic Control, vol. 62, no. 5, pp. 2546-2553, 2017.

[20] B.-Z. Guo and W. Guo, "The strong stabilization of a one-dimensional wave equation by non-collocated dynamic boundary feedback control," Automatica, vol. 45, no. 3, pp. 790-797, 2009.

[21] Z. J. Zhao, Z. J. Liu, Z. F. Li, N. Wang, and J. F. Yang, "Control design for a vibrating flexible marine riser system," Journal of The Franklin Institute, vol. 354, no. 18, pp. 8117-8133, 2017.

[22] K. D. Do, "Stochastic boundary control design for extensible marine risers in three dimensional space," Automatica, vol. 77, no. 3, pp. 184 197,2017

[23] U. H. Shah and K.-S. Hong, "Active vibration control of a flexible rod moving in water: Application to nuclear refueling machines," Automatica, vol. 93, pp. 231-243, 2018.

[24] H.-N. Wu and J.-W. Wang, "Static output feedback control via PDE boundary and ODE measurements in linear cascaded ODE-beam systems," Automatica, vol. 50, no. 11, pp. 2787-2798, 2014.

[25] F.-Y. Wang, N.-N. Zheng, D. Cao, C. M. Martinez, L. Li, and T. Liu, "Parallel driving in cpss: a unified approach for transport automation and vehicle intelligence," IEEE/CAA Journal of Automatica Sinica, vol. 4 no. 4, pp. 577-587, 2017.

[26] J.-M. Wang, B. Ren, and M. Krstic, "Stabilization and Gevrey regularity of a Schrödinger equation in boundary feedback with a heat equation," IEEE Transactions on Automatic Control, vol. 57, no. 1, pp. 179-185, 2012.

[27] B. Luo, H.-N. Wu, and H.-X. Li, "Adaptive optimal control of highly dissipative nonlinear spatially distributed processes with neuro-dynamic programming," IEEE Transactions on Neural Networks and Learning Systems, vol. 26, no. 4, pp. 684-696, 2015.

[28] J.-W. Wang, H.-N. Wu, and H.-X. Li, "Stochastically exponential stability and stabilization of uncertain linear hyperbolic PDE systems with Markov jumping parameters," Automatica, vol. 48, no. 3, pp. 569576, 2012.

[29] F.-Y. Wang, J. Zhang, Q. Wei, X. Zheng, and L. Li, "PDP: parallel dynamic programming," IEEE/CAA Journal of Automatica Sinica, vol. 4, no. 1, pp. 1-5, 2017.

[30] H.-N. Wu, H.-D. Wang, and L. Guo, "Disturbance rejection fuzzy control for nonlinear parabolic PDE systems via multiple observers," IEEE Transactions on Fuzzy Systems, vol. 24, no. 6, pp. 1334-1348, 2016.

[31] T. Endo, F. Matsuno, and Y. Jia, "Boundary cooperative control by flexible Timoshenko arms," Automatica, vol. 81, pp. 377-389, 2017.

[32] Z. Liu, J. Liu, and W. He, "Modeling and vibration control of a flexible aerial refueling hose with variable lengths and input constraint," Automatica, vol. 77, pp. 302-310, 2017.
[33] H.-N. Wu and H.-Y. Zhu, "Guaranteed cost fuzzy state observer design for semilinear parabolic PDE systems under pointwise measurements," Automatica, vol. 85, pp. 53-60, 2017.

[34] J.-M. Wang, J.-J. Liu, B. Ren, and J. Chen, "Sliding mode control to stabilization of cascaded heat PDE-ODE systems subject to boundary control matched disturbance," Automatica, vol. 52, pp. 23-34, 2015.

[35] D. Q. Huang, J. X. Xu, X. Li, C. Xu, and M. Yu, "D-type anticipatory iterative learning control for a class of inhomogeneous heat equations," Automatica, vol. 49, no. 8, pp. 2397-2408, 2013.

[36] X. Cai and M. Krstic, "Nonlinear stabilization through wave PDE dynamics with a moving uncontrolled boundary," Automatica, vol. 68, pp. 27-38, 2016.

[37] J. Dai and B. Ren, "Ude-based robust boundary control for an unstable parabolic PDE with unknown input disturbance," Automatica, vol. 93, pp. 363-368, 2018.

[38] T. Meng and W. He, "Iterative learning control of a robotic arm experiment platform with input constraint," IEEE Transactions on Industrial Electronics, vol. 65, no. 1, pp. 664-672, 2018.

[39] M. Krstic and A. Smyshlyaev, "Backstepping boundary control for firstorder hyperbolic PDEs and application to systems with actuator and sensor delays," Systems \& Control Letters, vol. 57, no. 9, pp. 750-758, 2008.

[40] H.-N. Wu, J.-W. Wang, and H.-X. Li, "Fuzzy boundary control design for a class of nonlinear parabolic distributed parameter systems," IEEE Transactions on Fuzzy Systems, vol. 22, no. 3, pp. 642-652, 2014.

[41] C. Xu, E. Schuster, R. Vazquez, and M. Krstic, "Stabilization of linearized 2D magnetohydrodynamic channel flow by backstepping boundary control," Systems \& Control Letters, vol. 57, no. 10, pp. 805812, 2008.

[42] X. He, W. He, J. Shi, and C. Sun, "Boundary vibration control of variable length crane systems in two dimensional space with output constraints," IEEE/ASME Transactions on Mechatronics, vol. 22, no. 5, pp. 19521962, 2017.

[43] J.-M. Wang, J.-J. Liu, B. Ren, and J. Chen, "Sliding mode control to stabilization of cascaded heat PDE-ODE systems subject to boundary control matched disturbance," Automatica, vol. 52, pp. 23-34, 2015.

[44] I. Karafyllis and M. Krstic, "Sampled-data boundary feedback control of 1-d parabolic pdes," Automatica, vol. 87, pp. 226-237, 2018.

[45] K.-J. Yang, K.-S. Hong, and F. Matsuno, "Energy-based control of axially translating beams: varying tension, varying speed, and disturbance adaptation," IEEE Transactions on Control Systems Technology, vol. 13, no. 6, pp. 1045-1054, 2005.

[46] T. Endo, F. Matsuno, and H. Kawasaki, "Simple boundary cooperative control of two one-link flexible arms for grasping," IEEE Transactions on Automatic Control, vol. 54, no. 10, pp. 2470-2476, 2009.

[47] L. Pavel and L. Chang, "Lyapunov-based boundary control for a class of hyperbolic lotka-volterra systems," IEEE Transactions on Automatic Control, vol. 57, no. 3, pp. 701-714, 2012.

[48] W. He, C. Sun, and S. S. Ge, "Top tension control of a flexible marine riser by using integral-barrier Lyapunov function," IEEE/ASME Transactions on Mechatronics, vol. 2, no. 20, pp. 497-505, 2015.

[49] Z. Liu, F. Wang, and Y. Zhang, "Adaptive visual tracking control for manipulator with actuator fuzzy dead-zone constraint and unmodeled dynamic," IEEE Transactions on Systems, Man, and Cybernetics: Systems, vol. 45, no. 10, pp. 1301-1312, 2015.

[50] Z. Liu, C. Chen, Y. Zhang, and C. L. P. Chen, "Adaptive neural control for dual-arm coordination of humanoid robot with unknown nonlinearities in output mechanism," IEEE Transactions on Cybernetics, vol. 45 , no. 3, pp. 507-518, 2015.

[51] C. L. Chen, G. X. Wen, Y. J. Liu, and Z. Liu, "Observer-based adaptive backstepping consensus tracking control for high-order nonlinear semistrict-feedback multiagent systems," IEEE Transactions on Cybernetics, vol. 46, no. 7, pp. 1591-1601, 2016.

[52] Q. Zhou, L. Wang, C. Wu, H. Li, and H. Du, "Adaptive fuzzy control for nonstrict-feedback systems with input saturation and output constraint," IEEE Transactions on Systems, Man, and Cybernetics: Systems, vol. 47, no. 1, pp. 1-12, 2017.

[53] Z. Li, S. Xiao, S. S. Ge, and H. Su, "Constrained multilegged robot system modeling and fuzzy control with uncertain kinematics and dynamics incorporating foot force optimization," IEEE Transactions on Systems, Man, and Cybernetics: Systems, vol. 46, no. 1, pp. 1-15, 2016.

[54] W. He, Z. Li, and C. L. P. Chen, "A survey of human-centered intelligent robots: issues and challenges," IEEE/CAA Jouirnal of Automatica Sinica, vol. 4, no. 4, pp. 602-609, 2017.

[55] S. Zhang, Y. Dong, Y. Ouyang, Z. Yin, and K. Peng, "Adaptive neural control for robotic manipulators with output constraints and uncertainties," IEEE Transactions on Neural Networks and Learning Systems, 2018, in press, DOI: 10.1109/TNNLS.2018.2803827. 
[56] Y.-J. Liu, S. Lu, and S. Tong, "Neural network controller design for an uncertain robot with time-varying output constraint," IEEE Transactions on Systems, Man, and Cybernetics: Systems, vol. 47, no. 8, pp. 20602068, 2017.

[57] H. Goldstein, Classical Mechanics. Massachusetts, USA: AddisonWesley, 1951.

[58] H. Benaroya, Mechanical Vibration: Analysis, Uncertainties and Control. CRC Press, 2004.

[59] B. V. E. How, S. S. Ge, and Y. S. Choo, "Active control of flexible marine risers," Journal of Sound and Vibration, vol. 320, pp. 758-776, 2009.

[60] O. M. Faltinsen, Sea Loads on Ships and Offshore Structures. Cambridge, UK: Cambridge University Press, 1990.

[61] W. He, S. S. Ge, and S. Zhang, "Adaptive boundary control of a flexible marine installation system," Automatica, vol. 47, no. 12, pp. 2728-2734, 2011.

[62] R. Skjetne, T. Fossen, and P. Kokotović, "Adaptive maneuvering, with experiments, for a model ship in a marine control laboratory," Automatica, vol. 41, no. 2, pp. 289-298, 2005.

[63] K. P. Tee and S. S. Ge, "Control of fully actuated ocean surface vessels using a class of feedforward approximators," IEEE Transactions on Control Systems Technology, vol. 14, pp. 750-756, 2006. 